\title{
Expression of remyelination-modulating genes in astrocytes are controlled by MALAT1 and Lnc-DC long non-coding RNAs
}

\author{
Shahrzad Askari ${ }^{1}$, Fatemeh Khani-Habibabadi ${ }^{1}$, Mehrdad Behmanesh ${ }^{1}$ *
}

This manuscript has been withdrawn by the authors as it was submitted and made public without the full consent of all the authors. Therefore, the authors do not wish this work to be cited as reference for the project. If you have any questions, please contact the corresponding author. 